induced left ventricular dysfunction should be undertaken to define the magnitude of the risk," say the authors of the report, which was published online on 23 July in Nature Medicine (www.nature.com/naturemedicine, doi: $10.1038 / \mathrm{nm} 1446$ )

The authors say that patients who are taking imatinib should be followed closely so that symptoms or signs of left ventricular dysfunction can be spotted.

Thomas Force, of Jefferson Medical College, Philadelphia, and colleagues reported on 10 people who developed severe congestive heart failure while being treated with imatinib, and they say that mice treated with the drug developed heart problems. None of the human patients died. But microscopic and biochemical analyses of hearts of humans and mice treated with imatinib indicate that the drug is toxic to cardiac cells.

No warnings have been issued by drug firms or governments, said Dr Force.

David Spurgeon Quebec

\section{US women}

\section{doctors lag behind men in publishing in top journals}

Despite increases in the number of women doctors in the United States over the past 40 years they are still under-represented as first or senior authors on papers published in leading journals, finds research in the New England Journal of Medicine (2006;355:281-7).

The observation that many papers in leading journals were written by men prompted the authors to wonder what message that sent to ambitious young women. "Publication in medica journals is an important measure of academic productivity. It is also highly emphasized in the academic promotion process and an important means by which the academic medical community communicates," they wrote.

The study looked at original articles published in 1970, 1980, 1990, 2000, and 2004 in the New England Journal of Medicine, $J A M A$, the Annals of Internal Medicine, the Annals of Surgery,
Obstetrics $\mathcal{E}^{\circ}$ Gynecology, and the Journal of Pediatrics.

The researchers were able to identify the sex of $99 \%$ of 7249 US doctors listed as first or senior authors on these papers.

Overall the percentage of female first or senior authors increased from $6 \%$ in 1970 to $29 \%$ in 2004 .

Janice Hopkins Tanne New York

\section{US national} academies of science panellists are accused of financial conflicts

Nearly one in five scientists on the study panels of the US National Academies of Science have direct financial ties to companies or industry groups with a direct stake in the outcome of the study, says a report by the Center for Science in the Public Interest. However, it does not say how or even whether these ties affect the recommendations made in those studies.

The report evaluated the ties of 320 panel members who served on 21 committees over the past two and a half years. It found that $18 \%$ of the panellists had such direct ties. More than 8000 scientists serve on more than 500 studies looked at by the national academies in a typical year.

Merrill Goozner, the report's author, called on the national academies to extend their definition of financial conflicts of interest beyond having any monetary ties within the last five years. Those who fail to disclose such links should be banned from participating on a study panel for three years, he said.

The report also seeks to expand the national academies' definition of balance to include ideological perspectives and not just technical expertise: if a panel member has worked for a conservative think tank, then someone from a liberal equivalent should also be appointed. Bob Roehr Washington

Ensuring Independence and Objectivity at the National Academies is available at http://cspinet.org/new/pdf.

\section{NICE advises a more personalised approach to postnatal care}

\author{
Rebecca Coombes London
}

A low rate of breast feeding and inconsistent healthcare advice to new mothers are targeted by new clinical guidelines on postnatal care for England and Wales.

The guidance, issued by the National Institute for Health and Clinical Excellence (NICE) sets out a standard of care that women and their new babies can expect from labour through to eight weeks after birth.

It recommends personalised care for mothers-in which an individual care plan would be drawn up soon after birth-and a move away from the more common "tick box" approach. The guideline also says that all care providers, whether in hospitals or primary care, should implement an externally evaluated, structured programme that encourages breast feeding. The programme should use as a minimum standard the "baby friendly initiative," a worldwide programme run by Unicef and the World Health Organization.

The rate of breast feeding in England is among the lowest in Europe. The baby friendly initiative has been successful in increasing the rate of breast feeding through training and supporting health professionals. However, in England only 10\% of babies are born in a unit that is accredited by the baby friendly initiative. London, in common with several other areas, has no accredited unit as yet.

Carmel Duffy, deputy programme director of the UK baby friendly initiative, said: "We can now really go out and promote the initiative, because NICE is saying this has been proven to work."

NICE has also produced an easy to use "quick reference guide" for healthcare professionals that presents the guideline in a straightforward format. For example, it details what the full examination of a newborn baby at 72 hours should consist of. It also deals with the signs and symptoms of potentially serious or life threatening conditions in the mother or baby.

David Elliman, a consultant in community child health at Great Ormond Street Hospital and Islington Primary Care Trust and a member of the guideline development group, said, "Quite often people are given inconsistent advice. What new parents don't need is to be told one thing by the GP, another by the midwife, and another by the health visitor."

The guideline also details the importance of educating parents in caring for themselves and their baby-for example, knowing what support networks exist in their area.

Routine Postnatal Care of Women and their Babies is available at www.nice.org.uk.

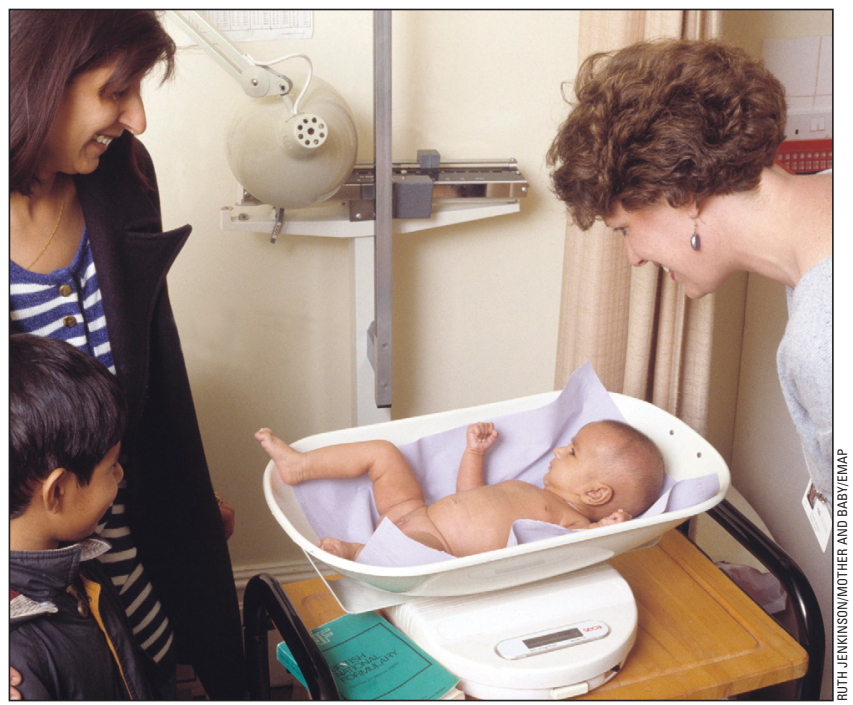

More personalised care is recommended in postnatal check-ups 